\title{
sciendo \\ PHOTOBIOLOGICAL SAFETY OF INCANDESCENT LAMP REPLACEMENTS
}

DOI 10.2478/ntpe-2018-0011

\author{
MSc. Eng. Andrzej Pawlak \\ Central Institute for Labour Protection - \\ National Research Institute, Poland
}

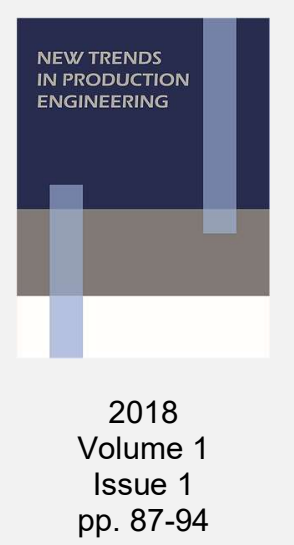

\begin{abstract}
The article, based on the records of the EN 62471:2008, presents guidelines for photobiological safety of lamps and lamp systems. There are allowed safe exposure times presented and emission limit values for each of four risk groups, defined by the standard. The paper presents criteria for lamps irradiance or radiance measurement. Discussed risks with association of ultraviolet, visible and infrared radiation. For a detailed presentation of the potential risks associated with the blue light, there is made a comparison of spectra of selected light sources. On these basis, the blue light hazard assessment was presented for selected light sources.
\end{abstract}

Keywords: Photobiological safety, risk groups, ultraviolet radiation, blue light hazard

\section{INTRODUCTION}

Withdrawing filament lamps from the lighting market across European Union states lead to their replacement, especially in households, with energy saving compact fluorescent lamps (integrated with electronic ignition systems), new generation halogen bulbs and high-power electroluminescence diodes (LEDs) in the form of single sources or modules. As per the schedule specified in the Directive (2005/32/EC), only the so-called alternative sources of light will remain in the market by 2016.

The main task of all sources of white light is to generate visible radiation with specific spectral characteristics. When selecting a light source for a specific visual task or a type of workstation, certain visible radiation parameters must be taken into account, such as: luminous flux, color rendering index or color temperature. The efficiency of the light source, related to its energy efficiency, is also an important parameter (Pawlak and Zalesińska, 2017). The remaining radiation components, such as ultraviolet and infrared radiation, are not desired and may even negatively affect the health and capability to work of the exposed person. Visible radiation with excessive amount of blue spectrum light has a similar impact on humans. However, the characteristics of radiation of the newly introduced lighting devices, especially of the LEDs, differs from that of the traditional incandescent sources due to a different way of generating light. Given the high number of producers of this sort of substitutes to filament sources (also originating from Asia), there are major differences of parameters of the optical radiation between similar types of light sources. Hence, a question arises whether the spectral characteristics of LED sources or compact fluorescent lamps (CFL) allows them to replace traditional light bulbs. These concerns are raised mainly by people whose eyes and skin are particularly sensitive to certain types of radiation, mainly ultraviolet and blue parts of the spectrum.

As not all sources of light are equally potentially harmful to health, a need arose to develop criteria of photobiological hazard caused by optical radiation emitted by these sources. The developed criteria of classification of hazard groups of lamps take into account the biological hazards caused by optical radiation specified in the Directive (2006/25/EC). They are covered in the standard EN (62471:2008). 


\section{CLASSIFICATION OF GROUPS OF HAZARDS OF LAMPS IN TERMS OF PHOTOBIOLOGICAL HAZARD}

The standard EN (62471:2008) was harmonized with the Directive (2006/95/EC) of the European Parliament and the Council on the harmonization of the laws of the Member States relating to the making available on the market of electrical equipment designed for use within certain voltage limits. The standard specifies photobiological safety criteria of optical radiation sources and lamp systems, as well as methods of measuring irradiation and radiance. According to the standard EN (62471: 2008) there are four hazard groups, defined as follows:

- free from hazard group (RG0) - lamps pose no photobiological hazard,

- $\quad$ hazard 1 group (low hazard) (RG1) - lamps pose no hazard in normal operating conditions,

- $\quad$ hazard 2 group (moderate hazard) (RG2) - lamps pose no hazard related to ocular reaction to very bright sources of light,

- $\quad$ hazard 3 group (high hazard) - lamps pose hazard even during brief exposures. It is not permitted to use such sources of light in general lighting.

This classification is based on maximum permissible exposure (MPE) assumed for the exposure of workers to hazards arising from artificial optical radiation, as specified in Directive $(2006 / 25 / E C)$ and takes into account the level of radiation emitted by a given device (a source of light or a lamp), the spectral range of radiation and access by humans. Based on the information specified in the standard EN (62471:2008), table 1 presents the relation between the type of photobiological hazard, the function of biological efficiency, the time of safe exposure with the emission limits of hazard groups for continuous operation lamps.

Table 1.

List of limit values of emission for continuous operation light sources, drawn up on the basis of the standard EN (62471: 2008)

\begin{tabular}{|c|c|c|c|c|c|c|c|c|c|c|}
\hline \multirow{2}{*}{$\begin{array}{l}\text { Type of } \\
\text { photobiolo- } \\
\text { gical hazard }\end{array}$} & \multirow{2}{*}{$\begin{array}{c}\text { Wave- } \\
\text { lengths } \\
\mathrm{nm}\end{array}$} & \multirow{2}{*}{$\begin{array}{l}\text { Function } \\
\text { of biolo- } \\
\text { gical } \\
\text { efficiency }\end{array}$} & \multirow{2}{*}{$\begin{array}{c}\text { Sym- } \\
\text { bol }\end{array}$} & \multirow[b]{2}{*}{$\begin{array}{c}\text { Denomi- } \\
\text { nation }\end{array}$} & \multicolumn{2}{|c|}{$\begin{array}{c}\text { Risk group } 0 \\
(\mathrm{RG}))\end{array}$} & \multicolumn{2}{|c|}{$\begin{array}{c}\text { Risk group } 1 \\
\text { (RG1) }\end{array}$} & \multicolumn{2}{|c|}{$\begin{array}{c}\text { Risk group } 2 \\
\text { (RG2) }\end{array}$} \\
\hline & & & & & $\begin{array}{l}\text { Safe } \\
\text { exp. } \\
\text { time }\end{array}$ & $\begin{array}{c}\text { Emission } \\
\text { limits }\end{array}$ & $\begin{array}{l}\text { Safe } \\
\text { exp. } \\
\text { time }\end{array}$ & $\begin{array}{c}\text { Emission } \\
\text { limits }\end{array}$ & $\begin{array}{l}\text { Safe } \\
\text { exp. } \\
\text { time }\end{array}$ & $\begin{array}{l}\text { Emission } \\
\text { limits }\end{array}$ \\
\hline Actinic UV & $\begin{array}{c}200 \div \\
400\end{array}$ & $S(\lambda)$ & $E_{s}$ & $w \cdot m^{-2}$ & $30000 \mathrm{~s}$ & 0.001 & $10000 s$ & 0.003 & $1000 \mathrm{~s}$ & 0.03 \\
\hline UV-A & $\begin{array}{c}315 \div \\
400\end{array}$ & - & Euva & $w \cdot m^{-2}$ & $1000 \mathrm{~s}$ & 10 & $300 \mathrm{~s}$ & 33 & $100 \mathrm{~s}$ & 100 \\
\hline Blue light & $\begin{array}{c}300 \div \\
700\end{array}$ & $B(\lambda)$ & $L_{B}$ & $\mathrm{Wm}^{-2} \mathrm{sr}^{-1}$ & $10000 \mathrm{~s}$ & 100 & $100 \mathrm{~s}$ & 10000 & $\left.0.25 \mathrm{~s}^{1}\right)$ & 4000000 \\
\hline $\begin{array}{l}\text { Retina } \\
\text { thermal } \\
\text { hazard }\end{array}$ & $\begin{array}{l}380 \div \\
1400\end{array}$ & $\mathrm{R}(\lambda)$ & $L_{R}$ & $\mathrm{Wm}^{-2} \mathrm{sr}^{-1}$ & $10 \mathrm{~s}$ & $28000 / \alpha$ & $10 \mathrm{~s}$ & $28000 / \alpha$ & $0.25 \mathrm{~s}^{1)}$ & $71000 / \alpha$ \\
\hline $\begin{array}{l}\text { Cornea and } \\
\text { lens } \\
\text { thermal } \\
\text { hazard }\end{array}$ & $\begin{array}{l}780 \div \\
3000\end{array}$ & - & $\begin{array}{l}E_{1} \\
R\end{array}$ & $\mathrm{~W} \cdot \mathrm{m}^{-2}$ & $1000 \mathrm{~s}$ & 100 & $100 \mathrm{~s}$ & 570 & $10 \mathrm{~s}$ & 3200 \\
\hline
\end{tabular}

$\alpha$ - angular subtense in radians; exp. = exposure

1) $0.25 \mathrm{~s}$ is accepted as the time of the defensive (aversive) reflex of the eyes

In the assumed classification, hazard 3 group (RG3) includes sources of light that may pose hazard even with temporary or brief exposure and whose parameter values, determined on the basis of measurements, serving to evaluate the hazard, exceed hazard 2 group's limits (RG2). The highest of the obtained hazard groups is assumed as the general hazard group of the examined lamp (resulting from all listed photobiological hazard).

Depending on the intended use of sources of light and luminaires, the following criteria of measuring the irradiation or radiance are applied in the standard EN (62471:2008):

- in case of sources for general lighting purposes used in e.g. offices, schools, apartments, production plants, on roads and in vehicles - the measurement should be made at a distance for which illuminance is equal to $500 \mathrm{~lx}$ (typical value of illuminance of general lighting used in offices, schools, etc.),

- in case of all other sources, including sources for special purposes such as displaying video images, reprographic processing, tanning bed salons, industrial processes, medical 
treatment and searching applications, the criterion is based on measuring the photobiological safety at a distance of $200 \mathrm{~mm}$ from the source of light.

Such a differentiation is of utmost importance, since e.g. in an office, nobody looks at a ceiling luminaire from the distance of $200 \mathrm{~mm}$, but in some industrial applications, e.g. on quality control stations, employees may have to look at the source of light from such a short distance. In such cases, additional instructions protecting the eyes from damage are necessary. One should also remember that if sources of light are placed in a luminaire, the hazard group classification may be changed by the luminaire's optics. Consequently, if the original parameters of the source of light are modified in any way by the luminaire, new measurements must be made to re-classify the luminaire.

The detailed requirements for measurement conditions and techniques of specific hazards are described in the standard EN (62471:2008) and in the publication (Pietrzykowski, 2012). The results of such evaluation for selected light sources and lighting fixtures are presented in the Guide (Publications Office of the EU, 2011).

\section{COMPARISON OF THE SPECTRA OF SELECTED BULB SUBSTITUTES}

This chapter compares the spectral characteristics of the main types of bulbs against compact fluorescent lamps and LED sources, in order to answer the questions posed in the introduction - whether the spectral characteristics of the two latter light sources allows them to replace traditional light bulbs. The comparison focuses on spectral radiation intensity in blue and ultraviolet range for various light sources, as opposed to radiation emitted by daylight (official CIE curve for daylight $6,500 \mathrm{~K}$ ) and by an incandescent source with an opal-colored bulb. In all cases the spectral radiation intensity measurements were performed so as to obtain light sources spectra at light intensity equal to $500 \mathrm{Ix}$ (as per the standard EN (62471:2008). A logarithmic scale was used to present the light sources measurement results, because the linear scale would fail to properly present the differences between various curves.

Figure 1 presents a comparison of radiation emitted by various LED sources (with E27 and GU10 stems and a halogen lamp substitute, MR16).

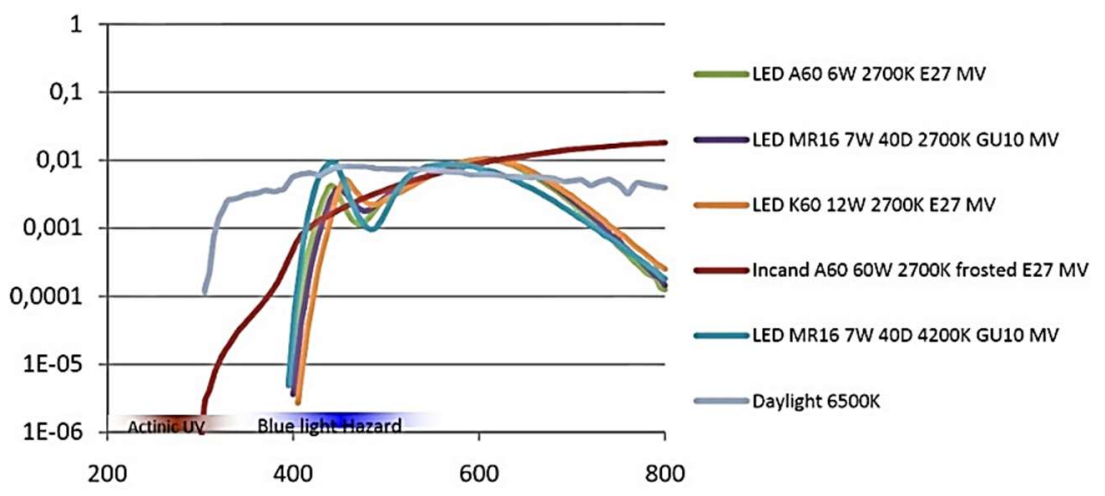

Fig. 1. Comparison of the characteristics of the spectral distribution of LEDs against

Source: CELMA, 2011 the spectrum of a light bulb and daylight

The maximal radiation of the LEDs emitting white light usually falls in the blue band (around 450 $\mathrm{nm}$ ) and a more broadband emission in the green and yellow band. A reduction can be seen near the blue maximal value, at approximately $490 \mathrm{~nm}$, which is also a part of the reaction curve of blue light. This reduction "compensates" the blue peak in LEDs, which is why the total emission of the blue light (presented as the area below the curve) emitted by the 2,700 K LED is comparable to the emission of the $2,700 \mathrm{~K}$ incandescent lamp.

Figure 2 presents the spectral distributions of two popular, energy saving fluorescent lamps (with exposed tubing - a burner, shielded with an opal-colored bulb - a bulb, with E27 socket) and their comparison to the spectrum of the bulb and daylight. A typical spectrum of a fluorescent lamp contains multiple maximal and minimal values. When determining the area below the curve for the purpose of determining blue radiation, it may be assumed that the maximal and minimal 
values balance out. It should be noted that the high maximal values are very narrow and consequently do not contribute to a large degree to the increased emission of blue light. On the left side, the spectral distribution curves slightly overlap the UV actinic reaction spectrum. Taking into account that the data are presented on a logarithmic scale, the UV actinic energy is very low and visibly lower than the natural daylight emission.

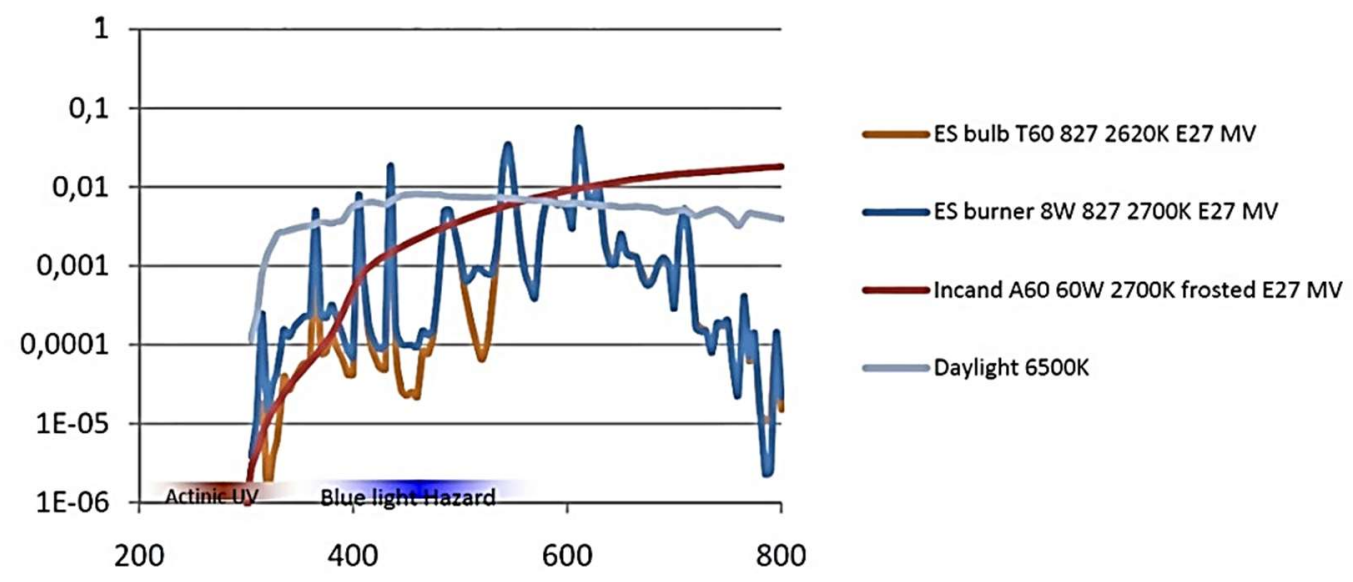

Fig. 2. Comparison of the characteristics of the spectral distribution of integrated, compact fluorescent lamps against the spectrum of a light bulb and daylight

Source: CELMA, 2011

The spectral distribution curves of halogen sources (Fig. 3) are shaped similarly as for the bulbs. They grow uniformly with the growth of the wavelength and descend in the infrared band. This makes halogen good alternatives for bulbs.
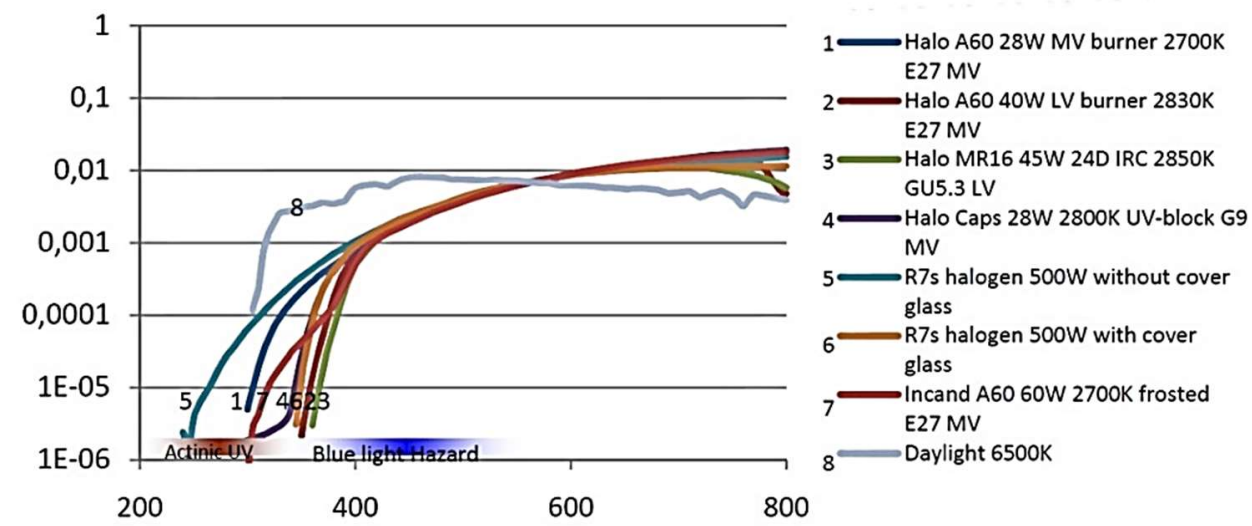

Fig. 3. Comparison of the characteristics of the spectral distribution of halogen lamps against the spectrum of a light bulb and daylight (CELMA, 2011): halogen lamps: 1, 2 - with A60 bulbs,

3 - an MR16 lamp with IR coat, 4 - a capsule with a UV filter, 5 - linear, without an additional lampshade in the luminaire, 6 - linear with a glass lampshade in the luminaire

The green line denotes a halogen lamp (no. 3) with an IR reduction coating (the curve drops visibly in the IR band). The violet curve (no. 4) shows that the UV quartz filter actually absorbs UV radiation efficiently, thanks to which the diagram approaches the bulb's curve. Other types of halogen sources exhibit lower UV emission than bulbs, especially the $500 \mathrm{~W}$ dual-stem sources (no. 6) which should be always used with proper glass lampshades. In such cases, the characteristics of these halogen sources is similar to the characteristics of the bulbs.

Despite the fact that the spectra of LEDs, compact fluorescent lamps, halogen lamps and bulbs have different shapes, the share of blue light radiation is not significantly different for these sources (if their color temperature is similar), designed in various technologies, and is always significantly lower than the share of ultraviolet and blue radiation in daylight. It should be noted that in most cases, in sources designed for general lighting purposes, the value of ultraviolet 
emission is significantly lower than the exposure limits specified in the standard EN (62471:2008).

\section{EVALUATION OF THE HAZARD CAUSED BY SELECTED LIGHT SOURCES}

On the basis of comparisons of the spectra of selected light sources presented in Chapter 3 and the potential hazards for humans, it must be concluded that the blue light hazard is the most dangerous one. Therefore, this chapter will evaluate it in finer details. Generally, two cases can be distinguished when evaluating the blue light hazard caused by various light sources: observing the illuminated objects and looking directly into a light source.

\section{Observing the illuminated objects}

In most cases, humans look at objects illuminated by daylight or by electric light sources. Thanks to the application of correctly selected lighting fixtures we can avoid direct visual contact with light sources - serving mainly to eliminate glare. Consequently, this arrangement may be deemed safe in most cases. In such situations the irradiance, which determines the radiant flux incident on a unit of area, is the most important parameter to be evaluated.

The spectral distributions of light sources may be used to calculate the effective irradiance $\left(E_{B}\right)$ within blue light, using the relative spectral efficiency curve of the photochemical hazard $B(\lambda)$ for retina, defined in the standard EN (62471:2008), among else. Figure 4 presents the $E_{B}$ values of different, selected types of electric light sources and the daylight. For better comparison with the daylight, the $E_{B}$ values were determined for $500 \mathrm{~lx}$. It can be seen from the diagram that all light sources with similar color temperature have very similar $E_{B}$ values.

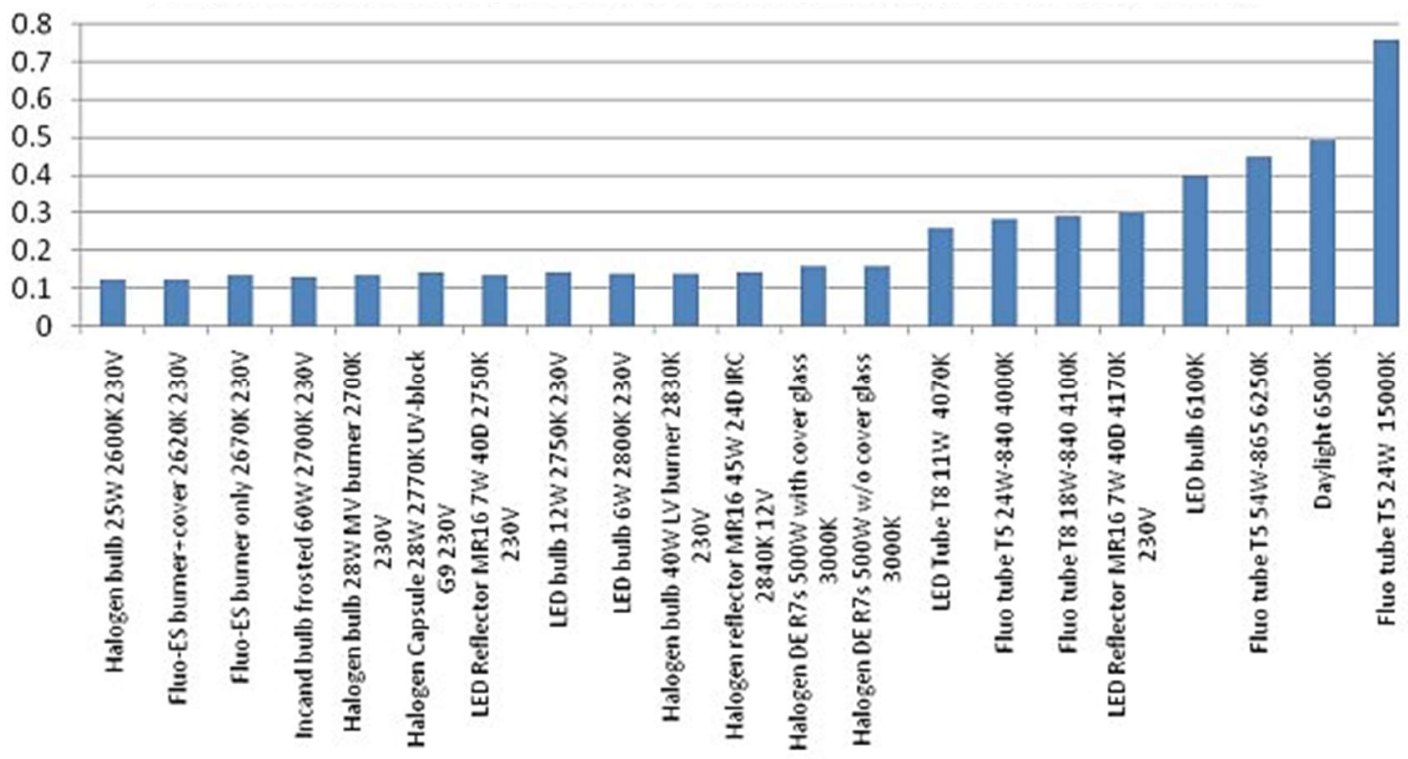

Fig. 4. A summary list of $E_{B}$ values of electric light sources and daylight Source: CELMA, 2011

In reality, the lighting intensity of daylight greatly exceeds $500 \mathrm{~lx}$. Therefore, Figure 5 presents $E_{B}$ values of selected light sources (determined at $500 \mathrm{~lx}$ ) and the daylight (determined at 5,000 $\mathrm{Ix}$ ), which constitutes an average value for moderate latitudes.

The practical lighting intensity for outdoor lighting may span a wide range of values, up to 50,000 Ix on a sunny day in moderated latitudes, reaching 100,000 Ix in tropical areas. This shows that the amount of blue light emitted by sources designed for general lighting purposes in rooms is low or very low when compared to outdoor conditions. 


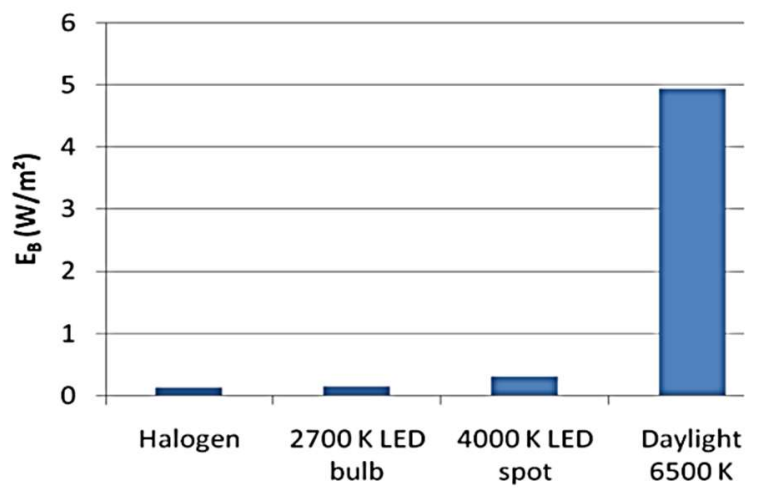

Fig. 5. A summary list of $E_{B}$ values of electric light sources $(500 \mathrm{~lx})$ and daylight $(5,000 \mathrm{~lx})$ Source: CELMA, 2011

\section{Looking at light sources directly}

The summary list of light sources for this case was also prepared according to the standard EN (62471:2008). The standard distinguishes small light sources, whose angular diameter $\alpha<11$ mrad. The image from a small source is blurred on the larger area of the retina due to controlled and uncontrolled eye movements, which reduces the number of photons falling onto a given point (receptor) of the retina and reduces the risk of damaging it (CELMA, 2011). In the summary list presented in Fig. 7 and 8, a more rigorous method applied for the remaining light sources was used, namely the measurement was done at a distance of $200 \mathrm{~mm}$, which is shorter than the distance at which lighting intensity is $500 \mathrm{~lx}$. Because most light sources cannot be treated as small sources at this distance, (effective) energetic luminance within blue radiation $\left(L_{B}\right)$ must be measured.

The luminous flux generated by linear fluorescent lamps (T8) is relatively big, but their luminance is relatively low - on the order of several dozen thousand $\mathrm{cd} / \mathrm{m}^{2}$ due to the large area of the source's lighting area. However, the coil of a halogen bulb emits luminous flux from a very small area, but its luminance is high, on the order of several or a dozen million $\mathrm{cd} / \mathrm{m}^{2}$. The luminance of the brightest LED source (module) is around ten million $\mathrm{cd} / \mathrm{m}^{2}$. For comparison, Sun's luminance is around 1 billion $\mathrm{cd} / \mathrm{m}^{2}$.

Figure 6 presents the values of effective energetic luminance (weighted according to the $B(\lambda)$ curve) of dispersed light sources with low luminance.

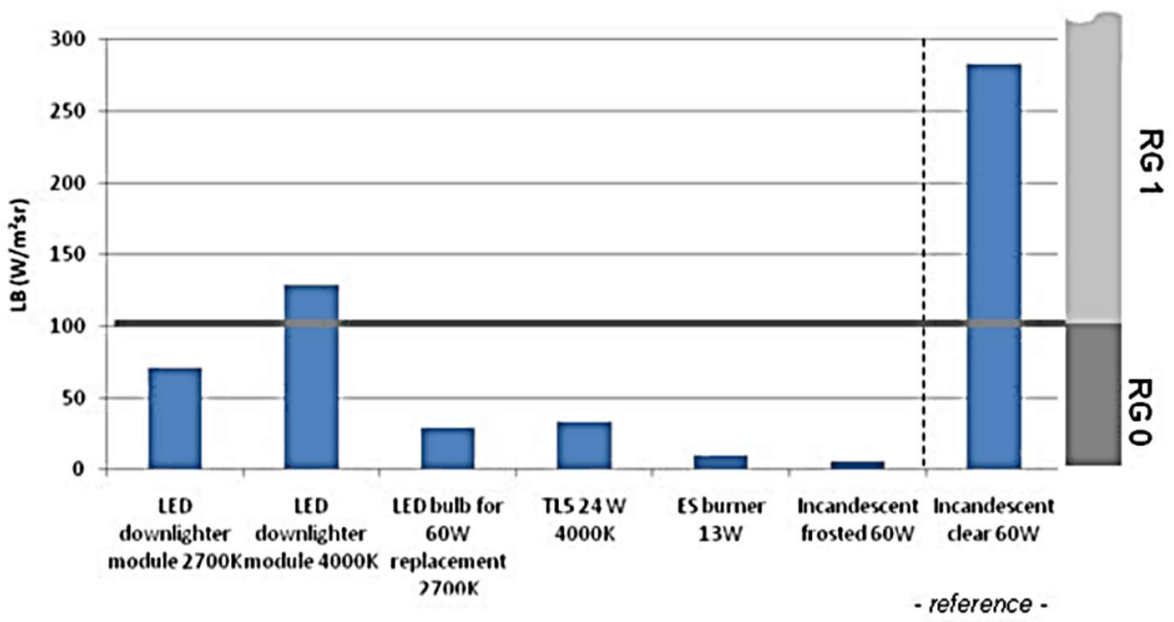

Fig. 6. Effective energetic luminance of blue radiation of example light sources with low luminance (CELMA, 2011): 2,700 K and 4,000 K LED modules;

LED source in a bulb $(2,700 \mathrm{~K})$ - a substitute for a $60 \mathrm{~W}$ bulb, a TL 5 fluorescent lamp rated at $24 \mathrm{~W}$ and 4,000 K, a compact fluorescent lamp with exposed tubing rated at $13 \mathrm{~W}$ and 2,700 K, a $60 \mathrm{~W}$ bulb - with a milk-colored bulb and with a transparent bulb - for reference 
Figure 7 presents the values of effective energetic luminance (weighted according to the $B(\lambda)$ curve) of point light sources with high luminance.

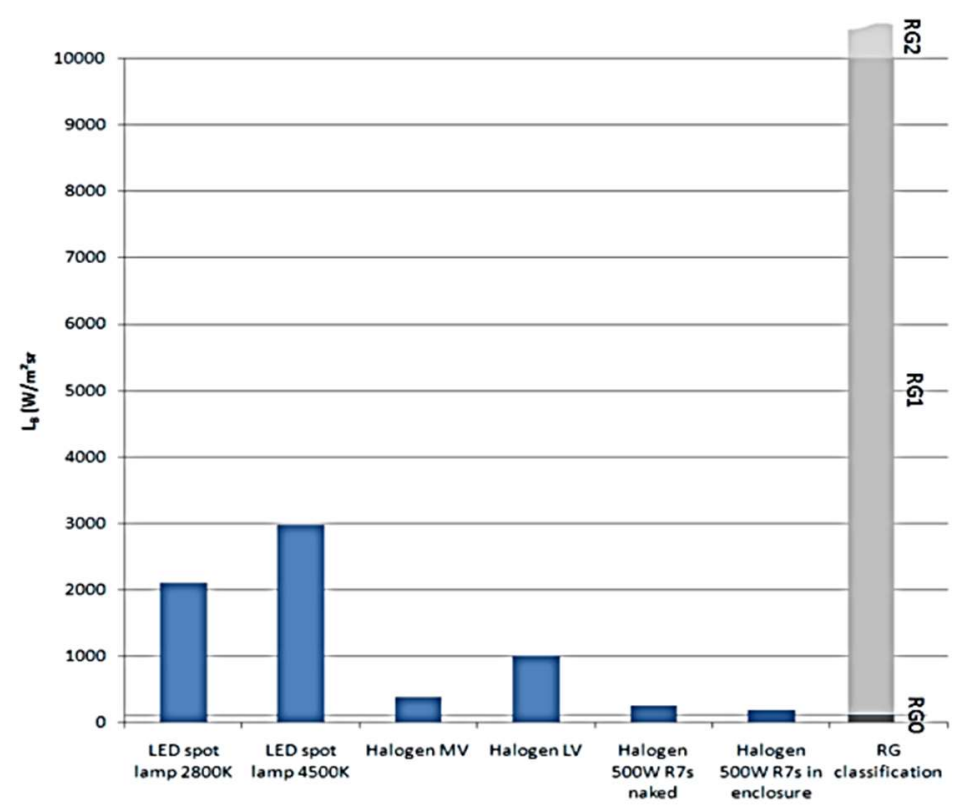

Fig. 7. Effective energetic luminance of blue radiation of example light sources with high luminance (CELMA, 2011): 2,800 K and 4,500 K LED modules as substitutes for halogen sources supplied with mains power or from low voltage installations, as well as $500 \mathrm{~W}$ linear halogen lamps, exposed and with additional glass lampshades

The energetic luminance of blue radiation of dispersed light sources is relatively low. Assigning sources of light to hazard groups (as per EN (62471:2008)) shows that most of them belong to the RG0 group. At higher color temperature values $(4,000 \mathrm{~K})$ some of them may be classified as RG1, with maximal exposure times exceeding 1 hour. It should be noted that this exposure time applies to looking directly at the source of light from a short distance. In normal operating conditions, when the eye's distance to the source is much higher than the value of $200 \mathrm{~mm}$ used for measurement purposes, these sources are safe.

All evaluated point light sources were classified as RG 1 category. Consequently, they do not pose any threat in normal operating conditions and require no special warning signs. Still, one should avoid looking directly at these sources, especially from a low distance. The maximal exposure times for the presented sources are 200 seconds or longer.

In both cases, however, such long exposure times will not be reached in practice, because humans involuntarily look away from highly glaring sources of light (instinctive aversion reaction).

\section{CONCLUSION}

Drawing from the analysis of the cited summary lists, a conclusion can be made that substitutes of the main series of bulbs in the form of LED sources (single sources and modules), integrated compact fluorescent lamps and halogen lamps are safe for users in terms of photobiological safety, when used as intended. For a given color temperature, the share of blue light in LED sources is no different than its share sources using other technologies. Despite that, one must avoid looking directly at bright, spot-like sources such as LEDs, transparent light bulbs, discharge sources or the Sun. Still, even when one randomly looks at a bright source of light, a natural reflex appears (the eye close or are averted involuntarily).

Workers often complain about various ailments, especially of the eyes, traceable to improper lighting of work stations, and more accurately, with the use of various types of replacement light bulbs. The use of replacement light bulbs is not always the source of complaints; rather, it is the use of poor quality replacement parts. Additionally, the used luminaires should be properly 
selected for the type of performed visual activity. For example, linear fluorescent lamps must not be substituted with high power LEDs installed inside glass tubing whose dimensions match those of a fluorescent lamp. Pursuant to the Directive (2006/25/EC), the workers' sight should be protected against visible radiation emitted by LEDs to the same extent as it should be protected against laser radiation. Consequently, every light emitting diode should have its own light and optical system.

\section{ACKNOWLEDGEMENT}

This paper has been prepared on the basis of the results of a research task carried out within the scope of the third stage of the National Programme "Improvement of safety and working conditions" supported in 2017-2019 - within the scope of state services and statutory activity by the Ministry of Labour and Social Policy. The Central Institute for Labour Protection - National Research Institute is the Programme's main co-ordinator.

\section{REFERENCES}

Pawlak A., Zalesińska M. (2017). Comparative study of light sources for household. Management Systems in Production Engineering, Volume 25, Issue 1, pp 35-41.

Pietrzykowski J. (2012). Metrological aspects of application of standard EN 62471 Photobiological safety of lamps and lamps systems. Proceedings of Elektrotechnical Institute, 255, pp 45-52.

Directive 2006/25/EC of the EEC of 5 April 2006 on the min. health and safety requirements regarding the exposure of workers to risks arising from physical agents (19th individual Directive within the meaning of Article 16(1) of Directive 89/391/EEC).

Directive 2005/32/EC of the EEC of 6 July 2005 establishing a framework for the setting of ecodesign requirements for energy-using products and amending Council Directive 92/42/EEC and Directives 96/57/EC and 2000/55/EC of the EEC.

Directive 2006/95/EC of the EEC of 12 December 2006 on the harmonization of Member States relating to electrical equipment designed for use within certain voltage limits.

EN 62471: 2008 Photobiological safety of lamps and lamp systems.

Non-binding guide to good practice for implementing Directive 2006/25/EC. "Artificial Optical Radiation", (2011). Publications Office of the European Union, Luxembourg.

Optical safety of LED lighting (2011). 1st $^{\text {st }}$ edition, July 2011 CELMA - European Lamps Companies Federation. 